\title{
Chemoradiation treatment patterns among United States Veteran Health Administration patients with unresectable stage III non-small cell lung cancer
}

Anna Hung ${ }^{1,2,3^{*}}$, Kyung Min Lee ${ }^{4}$, Julie A. Lynch ${ }^{4}$, Yanhong Li $i^{1,3}$, Pradeep Poonnen ${ }^{3,5}$, Olga V. Efimova ${ }^{4}$, Bradley J. Hintze ${ }^{1,3}$, Trudy Buckingham ${ }^{6}$, Candice Yong ${ }^{6}$, Brian Seal ${ }^{6}$, Michael J. Kelley ${ }^{3,5}$ and Shelby D. Reed ${ }^{1,2,3}$

\begin{abstract}
Background: The Veterans Health Administration (VHA) is the largest integrated health care system in the United States (US). Among VHA patients, the rate of use of concurrent chemoradiation therapy (CCRT) among those with unresectable, stage III non-small cell lung cancer (NSCLC) is unknown. The objective was to report recent CCRT treatment patterns in VHA patients and identify characteristics associated with receipt of CCRT.

Methods: Using Department of Veteran Affairs (VA) Cancer Registry System data linked to VA electronic medical records, we determined rates of CCRT, sequential CRT (SCRT), radiation therapy (RT) only, chemotherapy (CT) only, and neither treatment.

Results: Among 4054 VHA patients who met study criteria, CCRT rates slightly increased from 44 to 50\% between 2013 and 2017. Factors associated with decreased odds of CCRT receipt compared to any other treatment included increasing age (adjusted odds ratio [aOR] per 10 years $=0.67 ; 95 \% \mathrm{Cl}: 0.60-0.76)$ and Charlson-Deyo comorbidity score $(\mathrm{aOR}=0.94 ; 95 \% \mathrm{Cl}: 0.91-0.97)$. White race was associated with increased odds of CCRT receipt (aOR $=1.24$; $95 \%$ Cl: 1.004-1.53). In a chart review sample of 200 patients, less than half $(n=85)$ had a documented reason for not receiving CCRT. Among these, 29\% declined treatment, and 71\% did not receive CCRT due to "not being a candidate" for reasons related to frailty or lung nodules being too far apart for radiation therapy.

Conclusions: CCRT rates among VHA patients with unresectable, stage III NSCLC slightly increased from 2013 to 2017; however in 2017, only half were receiving CCRT. Older patients and those with multiple comorbidities were less likely to receive CCRT and even when controlling for these factors, non-white patients were less likely to receive CCRT.
\end{abstract}

Keywords: Non-small cell lung Cancer, Concurrent and sequential Chemoradiation, Veterans health administration

\footnotetext{
* Correspondence: anna.hung@duke.edu

'Duke Clinical Research Institute, Duke University School of Medicine, Durham, NC, USA

${ }^{2}$ Department of Population Health Sciences, Duke University School of Medicine, Durham, NC, USA

Full list of author information is available at the end of the article
}

(c) The Author(s). 2021 Open Access This article is licensed under a Creative Commons Attribution 4.0 International License, which permits use, sharing, adaptation, distribution and reproduction in any medium or format, as long as you give appropriate credit to the original author(s) and the source, provide a link to the Creative Commons licence, and indicate if changes were made. The images or other third party material in this article are included in the article's Creative Commons licence, unless indicated otherwise in a credit line to the material. If material is not included in the article's Creative Commons licence and your intended use is not permitted by statutory regulation or exceeds the permitted use, you will need to obtain permission directly from the copyright holder. To view a copy of this licence, visit http://creativecommons.org/licenses/by/4.0/ The Creative Commons Public Domain Dedication waiver (http://creativecommons.org/publicdomain/zero/1.0/) applies to the data made available in this article, unless otherwise stated in a credit line to the data. 


\section{Introduction}

In patients with unresectable stage III non-small cell lung cancer (NSCLC), high-level evidence from randomized controlled trials published starting in the 1990s have demonstrated that concurrent chemoradiation therapy (CCRT) results in improved overall survival compared to radiation therapy (RT) alone [1-4] or sequential chemoradiation therapy (SCRT) [5-9] with tolerable additional toxicity. However, guidelines also note that as part of the treatment selection process, one should consider a patient's ability to tolerate CCRT [10]. For example, CCRT has a higher rate of grade 3 or 4 esophagitis than SCRT [5]. As a result, frail patients may not be able to tolerate CCRT [10-12].

In the United States (US), military veterans (i.e., those who served in the armed forces), are eligible for medical care from the Veterans Health Administration (VHA). The VHA is the largest integrated health care system in the US [13]. It has approximately 171 medical centers and 1112 outpatient sites of care and serves approximately 9 million patients each year [13]. The majority of VHA patients are male, married, white, and non-Hispanic [14]. Compared to the general US population, VHA patients tend to be older, have lower levels of income and education, and have a higher comorbidity burden [15]. In 2010, $18 \%$ of incident veteran cancer cases diagnosed in the VHA were lung cancer [16]. Many VHA patients are current $(16 \%)$ or past $(61 \%)$ smokers, which can impact histology and treatment of NSCLC [14].

Between 2001 and 2010, only one-quarter of VHA patients with stage III NSCLC received chemotherapy and radiation within 4 months of diagnosis and had unresectable disease [17]. Among those patients who received chemotherapy (CT) and RT, almost $60 \%$ received CCRT (as opposed to SCRT). The primary objective of this study was to report on more recent nationwide CCRT treatment patterns in VHA patients and identify patientand facility-level factors associated with receipt of CCRT. The secondary objective was to report reasons why patients did not receive CCRT.

\section{Methods}

\section{Cohort identification}

VHA patients initially diagnosed with stage III NSCLC between January 1, 2013 and December 31, 2017 were identified using the VA Corporate Data Warehouse (CDW), which contains an extract of the VA Cancer Registry System (CRS). Patients were identified based on a primary cancer site of "lung/bronchus" in the VA CRS data and lung cancer diagnosis codes from the Ninth and Tenth Revisions of the International Classification of Diseases (Additional file 1: Appendix Table 1). Patients with NSCLC were retained based on histology codes (Additional file 1: Appendix Table 2). Patients were excluded if a stage IV NSCLC diagnosis was documented in VA CRS data within 1 month before or after the stage III diagnosis. This is because a documented diagnosis of stage IV before stage III was likely a medical record error, and a diagnosis of stage IV within 1 month after the diagnosis of stage III meant the patient would likely be treated for stage IV disease and CCRT would no longer be recommended.

VA CDW data were used to exclude patients who underwent lung resection in the 180 days following diagnosis (see procedure codes in Additional file 1: Appendix Tables 3a, 3b, and 3c) to identify those with unresectable stage III disease. To further limit the study population to patients who were receiving cancer care within the VHA, only patients who had at least two visits for cancer care in the 120 days following diagnosis were included. To ensure these visits were related to cancer care, patients had to have at least two visits to clinics related to cancer care based on clinic 'stop' codes (Additional file 1: Appendix Table 4) or at least two clinical notes that mentioned 'lung cancer'. Next, National Death Index data were used to exclude patients who died within 45 days of diagnosis to avoid a bias towards underestimating treatment rates. Lastly, to account for receipt of chemotherapy and/or radiotherapy in nonVHA settings documented by local cancer registrars, only patients whose VA CRS abstract status was complete were included.

\section{Treatment definitions}

We based our initial treatment definitions on a previous VA study, examining CT and RT within 120 days of diagnosis [17]. Chemotherapy (CT), based on the drugs reported in Additional file 1: Appendix Table 5, and RT, based on the procedure codes in Additional file 1: Appendix Table 6, that occurred within 120 days of diagnosis were identified. "CCRT" was defined as CT and RT that started within 14 days of each other. "SCRT" was defined as the receipt of CT and RT within 120 days of diagnosis that did not start within 14 days of each other. "CT only" was defined as CT alone within 120 days of diagnosis, and "RT only" was defined as RT alone within 120 days of diagnosis. "Neither treatment" was defined as not having received CT or RT within 120 days of diagnosis.

To assess the accuracy of applying these definitions to data from the VA CRS and CDW, 200 charts were randomly selected for review to assess concordance. Among those who did not receive CCRT, reasons for not receiving CCRT, if documented, were abstracted.

We performed three sensitivity analyses. First, we lengthened the time window used to define initial treatment from 120 days to 180 days of cancer diagnosis. Second, we varied the 14-day time window to define CCRT 
to 7 days, 21 days, and 30 days, since past studies have used different time windows $[17,18]$. Third, given concerns about underreporting of radiation therapy services received outside of $\mathrm{VHA}$, we narrowed the cohort to patients receiving care at VHA facilities equipped to provide on-site radiation therapy services.

\section{Patient- and facility-level characteristics}

Patient- and facility-level characteristics were derived from CDW and VA CRS data corresponding to the initial cancer diagnosis year unless otherwise noted. These included sociodemographic characteristics such as age, gender, race, ethnicity, marital status, Medicare enrollment at any point between 2013 and 2017, Medicaid eligibility, VHA priority status, rurality of patient residence, distance between patient residence and VA medical center, histology, smoking status, and Charlson-Deyo comorbidity score. In the US, Medicare and Medicaid are other health insurance programs generally for older adults and those with lower incomes, respectively $[19,20]$. Since VHA patients can also have additional insurance coverage such as through Medicare and Medicaid, it is important to account for these characteristics since additional insurance coverage can affect treatment. VHA priority groups are also important to account for when examining VHA care because they determine copay (i.e., costs that patients pay for health care) levels and how soon after military service patients are eligible for health care benefits, which can also affect treatment $[21,22]$. Generally, a disability that is highly connected to military service (e.g., $\geq 50 \%$ ) leads to assignment to a higher priority group with no copay requirements [21]. Other factors also impact priority group assignment. Such factors include income level and military service during specific time periods [21].

In addition to patient-level characteristics, facility-level characteristics were examined. These included whether the medical center had been certified by Commission on Cancer, geographic region, total number of unique patients seen at oncology clinics, and total number of oncologists at the medical center. In the US, the Commission on Cancer establishes standards to ensure quality, multidisciplinary, and comprehensive cancer care delivery in health care settings and has certified more than 1500 programs [23]. Geographic regions were measured as Census regions, which are regions set by the United States Census Bureau framework for grouping states to allow for consistency across time and studies [24]. The aforementioned patient- and facilitylevel characteristics were examined and adjusted for in models because all could be associated with receipt of CCRT.

\section{Statistical analysis}

Descriptive statistics were provided for receipt of CCRT, SCRT, RT only, CT only, and neither treatment overall, by calendar year, and by Census region. In addition, variations in treatment patterns were also examined across VHA facilities. Bivariate associations between a given patient or facility characteristic and the treatment type were analyzed using Chi-square tests for categorical variables and Kruskal-Wallis tests for continuous variables.

Generalized linear mixed models using binomial distributions and logit links and accounting for clustering by VA facility were used to identify factors independently associated with CCRT receipt compared to other treatment (SCRT, CT only, or RT only). Statistical significance was defined at an alpha of 0.05. All analyses were performed in SAS 9.2 (Cary, NC).

The study was approved by the institutional review boards at Duke University, Durham VA, and VA Salt Lake City.

\section{Results \\ Study population}

Between January 1, 2013 and December 31, 2017, we identified 6414 VHA patients with stage III NSCLC. After excluding those with stage IV disease within 1 month of stage III diagnosis $(n=10)$, then those who underwent a lung resection procedure $(n=1026)$, then those who were not receiving their cancer care within VHA $(n=1191)$, then those who died within 45 days $(n=92)$, and then those who had incomplete registry records $(n=41)$, our final study population consisted of 4054 patients (Fig. 1).

\section{Treatment rates}

Forty-seven percent of patients received CCRT (Fig. 2). This rate fluctuated between 44 and $50 \%$ annually between 2013 and 2017, and between 42 and 51\% across geographic regions (Figs. 2 and 3). The rate also varied by medical center. Across the 108 medical centers, the mean and median CCRT rates were 45 and $47 \%$, respectively, with an interquartile range of 34 to $56 \%$ (Fig. 4, Additional file 1: Appendix Table 7). Rates of SCRT, RT only, CT only, and neither treatment varied between 10 and $20 \%$ overall, as well as by year and geographic region (Figs. 2 and 3). Similarly, mean and median rates of SCRT, RT only, CT only, and neither treatment across the medical centers varied between 10 and 16\% (Fig. 4, Additional file 1: Appendix Table 7).

In our chart review of 200 randomly selected records, we found $90 \%$ concordance between our use of structured data to identify treatments received versus manual review employing the same treatment definitions. Sensitivity analyses showed that treatment rates minimally changed when extending the initial treatment time 

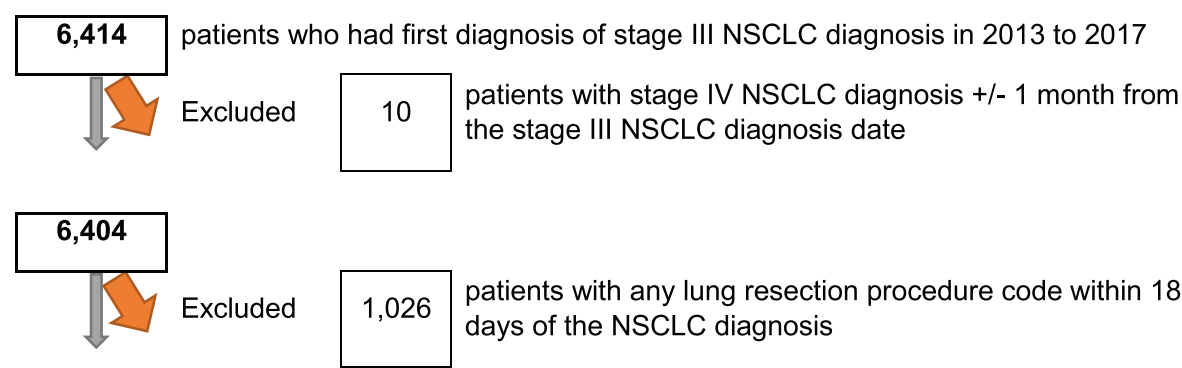

patients with any lung resection procedure code within 180 days of the NSCLC diagnosis
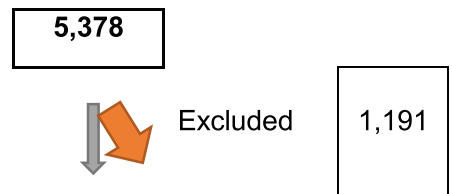

patients who did not have 2+ visits to VA for cancer care in the 120 days following the initial diagnosis (at least 2 stop codes or at least 2 clinical notes)

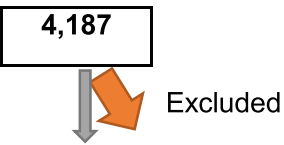

92 patients who died within 45 days of the initial diagnosis

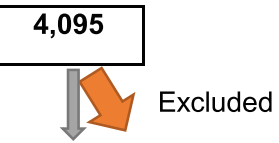

41 patients whose data had not been completely abstracted by a local cancer registrar Final Population

Fig. 1 Cohort identification

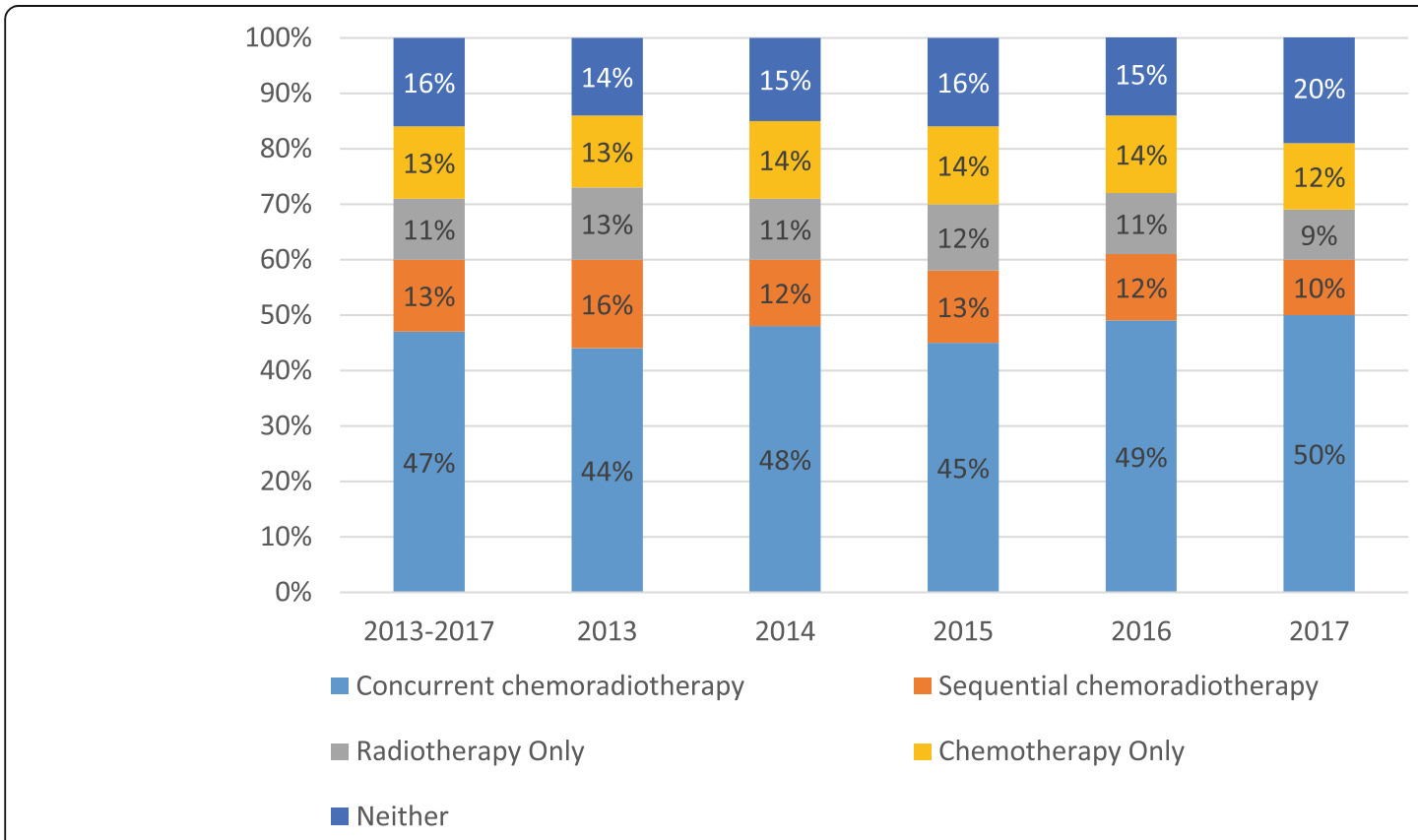

Fig. 2 Total and annual treatment rates in the full study population $(n=4054)$ 


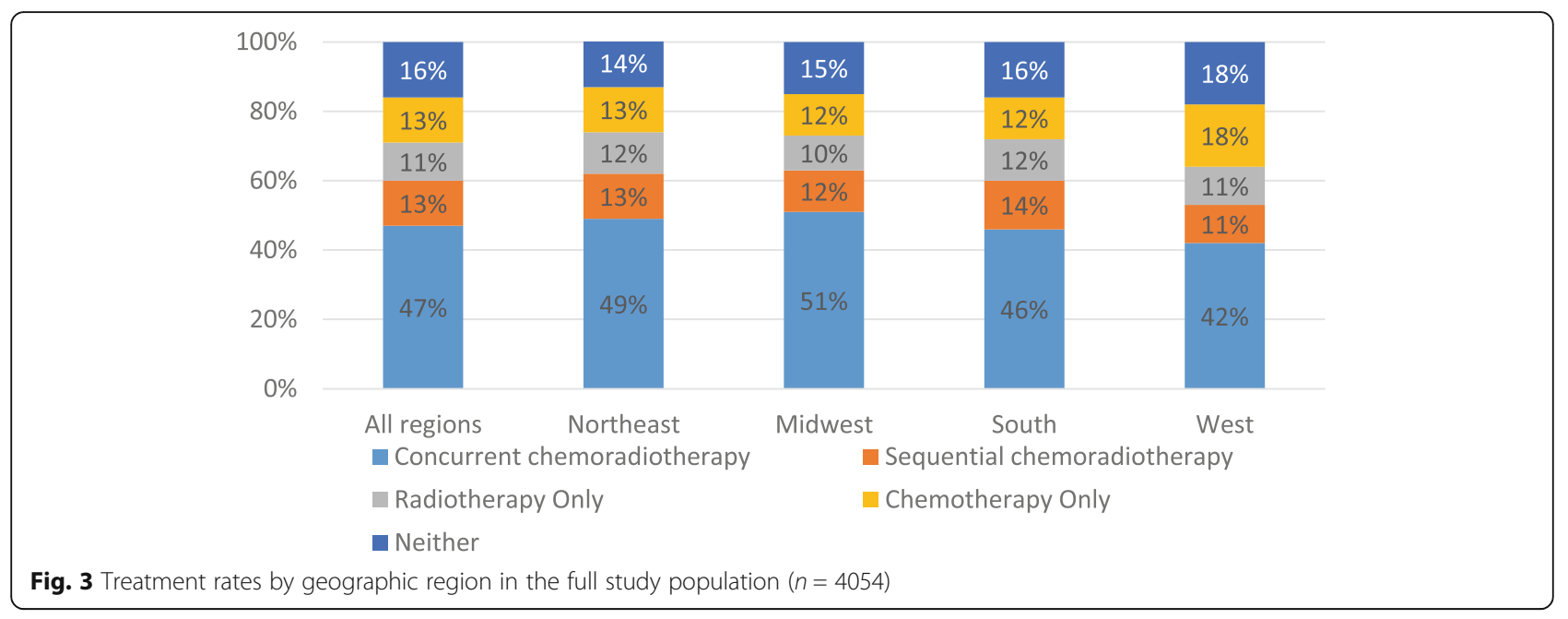

window from 120 days to 180 days (Additional file 1 : Appendix Table 8), differed more when varying the CCRT definition as CT and RT within 14 days to 7, 21, and 30 days (Additional file 1: Appendix Table 9), and minimally changed when restricting the population to patients who were seen at medical centers that provided on-site radiation therapy services (Additional file 1: Appendix Figs. 1-3).

\section{Patient and facility characteristics associated with receipt of CCRT}

The overall study population had a mean age of 68.7 years, and was predominantly male $(97.5 \%)$, white (78.0\%), and non-Hispanic (94.4\%; Table 1). The majority were enrolled in Medicare (82.9\%), few were eligible for Medicaid (1.7\%), and the mean Charlson-Deyo comorbidity score was 3.4. Approximately half had a nonservice connected disability (49.9\%), were married (44.6\%), and lived in an urban setting (62.4\%), with a

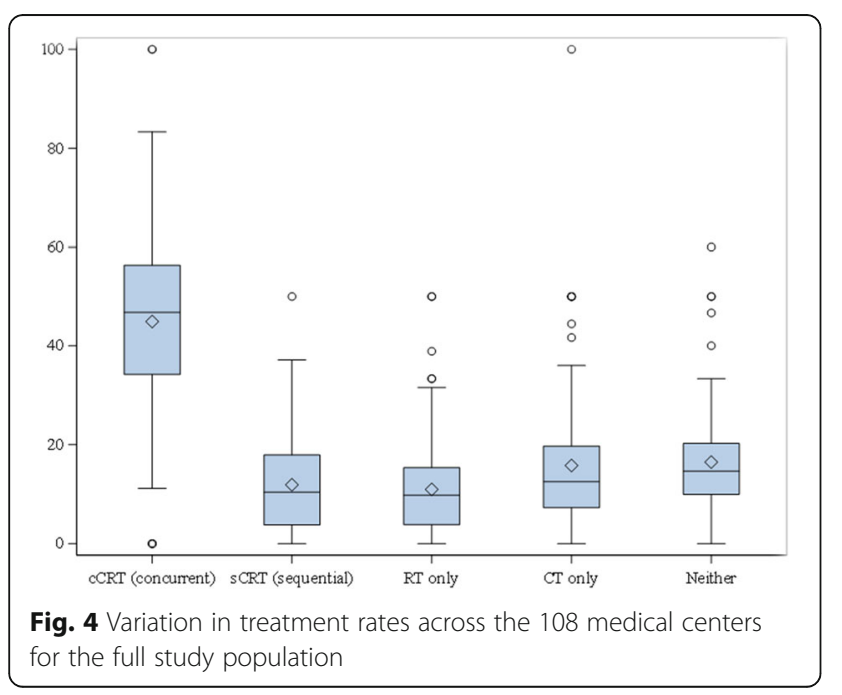

median distance between their residence and the medical center of 66.3 miles. Close to half of patients (51.9\%) had squamous cancer, and most were current $(56.0 \%)$ or former (34.5\%) smokers. Almost three-quarters (72.2\%) were seen at a medical center that was certified by Commission on Cancer, $44.5 \%$ were seen in the South, and the median numbers of oncology patients seen and oncologists working at medical centers during the diagnosis year were 196.0 and 12.0, respectively.

Among the 3414 patients who received treatment (i.e., CCRT, SCRT, RT only, or CT only), factors associated with increased odds of receipt of CCRT compared to any other treatment included white race (adjusted OR $[\mathrm{aOR}]=1.24 ; 95 \% \mathrm{CI}: 1.00-1.53$ ) and later diagnosis year (2017 vs 2013: $\mathrm{aOR}=1.65 ; 95 \%$ CI: $1.27-2.16 ; 2016$ vs 2013: aOR $=1.36 ; 95 \%$ CI: 1.06-1.76). Factors associated with decreased odds of CCRT receipt compared to any other treatment included increasing age (aOR per 10 years $=0.67 ; 95 \%$ CI: $0.60-0.76)$ and Charlson-Deyo comorbidity score (aOR $=0.94 ;$ 95\% CI: 0.91-0.97; Table 2).

\section{Chart review and reasons for not receiving CCRT}

Among the 200 charts reviewed, 142 patients did not receive CCRT. Of these, no reason was documented in electronic medical records for 57 patients (40\%; Additional file 1: Appendix Table 10). Of the 85 patients with a documented reason, $25(29 \%)$ patients declined treatment and 60 (71\%) were not considered candidates for CCRT. Of these, $40 \%$ were documented as too frail and $23 \%$ were considered to have disease that was too extensive or had lung nodules too far apart for radiation therapy. The remainder had no further information provided or were not a candidate due to comorbidities, disease progression/multiple cancers, or age (cell sizes too small to report). 
Table 1 Patient and medical center characteristics in all veterans and by treatment group

\begin{tabular}{|c|c|c|c|c|c|c|c|}
\hline Characteristics & $\begin{array}{l}\text { All patients } \\
(N=4054)\end{array}$ & $\begin{array}{l}\text { CCRT } \\
(N=1893)\end{array}$ & $\begin{array}{l}\text { SCRT } \\
(N=514)\end{array}$ & $\begin{array}{l}R T \text { only } \\
(N=466)\end{array}$ & $\begin{array}{l}C T \text { only } \\
(N=541)\end{array}$ & $\begin{array}{l}\text { Neither } \\
(N=640)\end{array}$ & $\underset{a}{P \text {-value }}$ \\
\hline \multicolumn{8}{|l|}{ Patient-related } \\
\hline \multicolumn{8}{|l|}{ Age } \\
\hline Mean (SD) & $68.7(8.2)$ & $67.3(7.4)$ & $67.4(7.3)$ & $72.3(8.9)$ & $69.0(8.4)$ & $71.4(8.9)$ & \\
\hline Median (Q1-Q3) & $68.0(63.0-74.0)$ & $67.0(63.0-71.0)$ & $67.0(63.0-71.0)$ & $71.0(66.0-80.0)$ & $67.0(63.0-75.0)$ & $70.0(65.0-78.0)$ & $<.0001$ \\
\hline \multicolumn{8}{|l|}{ Gender } \\
\hline Male & 3954 (97.5\%) & 1847 (97.6\%) & $496(96.5 \%)$ & $457(98.1 \%)$ & $530(98.0 \%)$ & $624(97.5 \%)$ & 0.5120 \\
\hline \multicolumn{8}{|l|}{ Race } \\
\hline White & $3164(78.0 \%)$ & 1499 (79.2\%) & $392(76.3 \%)$ & $343(73.6 \%)$ & $443(81.9 \%)$ & 487 (76.1\%) & 0.0053 \\
\hline Black or African American & $669(16.5 \%)$ & $304(16.1 \%)$ & $90(17.5 \%)$ & $88(18.9 \%)$ & 68 (12.6\%) & $119(18.6 \%)$ & \\
\hline Other & $67(1.7 \%)$ & $21(1.1 \%)$ & $15(2.9 \%)$ & + & $13(2.4 \%)$ & + & \\
\hline Unknown & $154(3.8 \%)$ & $69(3.6 \%)$ & $17(3.3 \%)$ & ++ & $17(3.1 \%)$ & ++ & \\
\hline \multicolumn{8}{|l|}{ Ethnicity } \\
\hline Hispanic or Latino & $97(2.4 \%)$ & $36(1.9 \%)$ & $13(2.5 \%)$ & $11(2.4 \%)$ & $22(4.1 \%)$ & $15(2.3 \%)$ & 0.0310 \\
\hline Not Hispanic or Latino & $3826(94.4 \%)$ & $1803(95.2 \%)$ & $490(95.3 \%)$ & $440(94.4 \%)$ & $493(91.1 \%)$ & $600(93.8 \%)$ & \\
\hline Unknown & $131(3.2 \%)$ & $54(2.9 \%)$ & $11(2.1 \%)$ & $15(3.2 \%)$ & $26(4.8 \%)$ & $25(3.9 \%)$ & \\
\hline \multicolumn{8}{|l|}{ Marital Status ${ }^{b}$} \\
\hline Married & $1810(44.6 \%)$ & ++ & ++ & ++ & ++ & ++ & 0.2897 \\
\hline Not Married & $2228(55.0 \%)$ & $1014(53.6 \%)$ & $281(54.7 \%)$ & $259(55.6 \%)$ & $290(53.6 \%)$ & $384(60.0 \%)$ & \\
\hline Unknown & $16(0.4 \%)$ & + & + & + & + & + & \\
\hline \multicolumn{8}{|c|}{ Medicare enrollment any time between 2013 and 2017} \\
\hline Yes & 3361 (82.9\%) & $1522(80.4 \%)$ & $426(82.9 \%)$ & $414(88.8 \%)$ & $441(81.5 \%)$ & $558(87.2 \%)$ & $<.0001$ \\
\hline \multicolumn{8}{|l|}{ Medicaid-eligible } \\
\hline Yes & $68(1.7 \%)$ & $27(1.4 \%)$ & + & + & ++ & + & 0.4563 \\
\hline No & 3910 (96.4\%) & 1835 (96.9\%) & $491(95.5 \%)$ & $448(96.1 \%)$ & $517(95.6 \%)$ & $619(96.7 \%)$ & \\
\hline Unknown & $76(1.9 \%)$ & $31(1.6 \%)$ & ++ & ++ & + & ++ & \\
\hline \multicolumn{8}{|l|}{ Priority status } \\
\hline Non-service connected & $2021(49.9 \%)$ & $917(48.4 \%)$ & $251(48.8 \%)$ & $262(56.29 \%)$ & $258(47.7 \%)$ & $333(52.0 \%)$ & 0.0553 \\
\hline Service connected < 50\% & $596(14.7 \%)$ & $269(14.2 \%)$ & $77(15.0 \%)$ & $68(14.6 \%)$ & $85(15.7 \%)$ & $97(15.2 \%)$ & \\
\hline $\begin{array}{l}\text { Service connected } 50 \text { to } \\
100 \%\end{array}$ & 1437 (35.4\%) & $707(37.3 \%)$ & $186(36.2 \%)$ & $136(29.2 \%)$ & $198(36.6 \%)$ & $210(32.8 \%)$ & \\
\hline \multicolumn{8}{|l|}{ Rurality of patient residence } \\
\hline Urban & $2531(62.4 \%)$ & $1178(62.2 \%)$ & $319(62.1 \%)$ & $310(66.5 \%)$ & $318(58.8 \%)$ & $406(63.4 \%)$ & 0.3908 \\
\hline \multicolumn{8}{|c|}{ Distance between patient residence and medical center } \\
\hline Mean (SD) & $93.2(122.4)$ & $85.9(85.9)$ & $88.6(143.3)$ & $83.9(76.1)$ & $114.5(185.2)$ & $107.2(150.5)$ & \\
\hline Median (Q1-Q3) & $\begin{array}{l}66.3(20.6- \\
127.5)\end{array}$ & $\begin{array}{l}64.4(20.5- \\
122.1)\end{array}$ & $\begin{array}{l}56.9(18.6- \\
113.6)\end{array}$ & $\begin{array}{l}66.1(18.2- \\
130.0)\end{array}$ & $\begin{array}{l}71.7(27.0- \\
131.4)\end{array}$ & $\begin{array}{l}74.4(21.2- \\
145.4)\end{array}$ & 0.0061 \\
\hline Unknown & + & + & + & + & + & + & \\
\hline \multicolumn{8}{|l|}{ Histology } \\
\hline Squamous & $2102(51.9 \%)$ & $1027(54.3 \%)$ & $291(56.6 \%)$ & $260(55.8 \%)$ & $229(42.3 \%)$ & $295(46.1 \%)$ & $<.0001$ \\
\hline Adenocarcinoma & $1447(35.7 \%)$ & $656(34.7 \%)$ & $171(33.3 \%)$ & $141(30.3 \%)$ & $236(43.6 \%)$ & $243(38.0 \%)$ & \\
\hline Other & $505(12.5 \%)$ & $210(11.1 \%)$ & $52(10.1 \%)$ & $65(13.9 \%)$ & 76 (14.0\%) & $102(15.9 \%)$ & \\
\hline
\end{tabular}


Table 1 Patient and medical center characteristics in all veterans and by treatment group (Continued)

\begin{tabular}{|c|c|c|c|c|c|c|c|}
\hline Characteristics & $\begin{array}{l}\text { All patients } \\
(N=4054)\end{array}$ & $\begin{array}{l}\text { CCRT } \\
(N=1893)\end{array}$ & $\begin{array}{l}\text { SCRT } \\
(N=514)\end{array}$ & $\begin{array}{l}R T \text { only } \\
(N=466)\end{array}$ & $\begin{array}{l}C T \text { only } \\
(N=541)\end{array}$ & $\begin{array}{l}\text { Neither } \\
(N=640)\end{array}$ & $\underset{a}{P \text {-value }}$ \\
\hline \multicolumn{8}{|l|}{ Smoking status } \\
\hline Current smoker & $2272(56.0 \%)$ & $1127(59.5 \%)$ & $299(58.2 \%)$ & $243(52.2 \%)$ & $266(49.2 \%)$ & $337(52.7 \%)$ & 0.0003 \\
\hline Former smoker & $1400(34.5 \%)$ & $619(32.7 \%)$ & $165(32.1 \%)$ & $176(37.8 \%)$ & $202(37.3 \%)$ & $238(37.2 \%)$ & \\
\hline Never smoker & $133(3.3 \%)$ & $47(2.5 \%)$ & $15(2.9 \%)$ & $17(3.7 \%)$ & $29(5.4 \%)$ & $25(3.9 \%)$ & \\
\hline Unknown & $249(6.1 \%)$ & $100(5.3 \%)$ & $35(6.8 \%)$ & $30(6.4 \%)$ & $44(8.1 \%)$ & $40(6.3 \%)$ & \\
\hline \multicolumn{8}{|c|}{ Charlson-Deyo comorbidity score } \\
\hline Mean (SD) & $3.4(2.6)$ & $3.2(2.4)$ & $3.3(2.6)$ & $4.0(2.9)$ & $3.3(2.6)$ & $3.6(2.6)$ & \\
\hline Median (Q1-Q3) & $3.0(1.0-5.0)$ & $3.0(1.0-4.0)$ & $3.0(2.0-4.0)$ & $3.0(2.0-6.0)$ & $3.0(1.0-4.0)$ & $3.0(2.0-5.0)$ & $<.0001$ \\
\hline 0 & $333(8.2 \%)$ & $167(8.8 \%)$ & ++ & ++ & ++ & ++ & $<.0001$ \\
\hline 1 & $684(16.9 \%)$ & $352(18.6 \%)$ & $76(14.8 \%)$ & $61(13.1 \%)$ & $83(15.3 \%)$ & $112(17.5 \%)$ & \\
\hline 2 & $676(16.7 \%)$ & $315(16.6 \%)$ & $94(18.3 \%)$ & $67(14.4 \%)$ & $106(19.6 \%)$ & $94(14.7 \%)$ & \\
\hline 3 & $760(18.7 \%)$ & $366(19.3 \%)$ & $109(21.2 \%)$ & $80(17.2 \%)$ & $96(17.7 \%)$ & $109(17.0 \%)$ & \\
\hline $4+$ & $1565(38.6 \%)$ & $675(35.7 \%)$ & $185(36.0 \%)$ & $225(48.3 \%)$ & $197(36.4 \%)$ & $283(44.2 \%)$ & \\
\hline Unknown & $36(0.9 \%)$ & $18(1.0 \%)$ & + & + & + & + & \\
\hline \multicolumn{8}{|c|}{ Medical Center-related } \\
\hline \multicolumn{8}{|c|}{ Certified by Commission on Cancer } \\
\hline Yes & $2928(72.2 \%)$ & 1379 (72.8\%) & $387(75.3 \%)$ & $354(76.0 \%)$ & $376(69.5 \%)$ & $432(67.5 \%)$ & 0.0162 \\
\hline No & 1107 (27.3\%) & $503(26.6 \%)$ & ++ & ++ & ++ & ++ & \\
\hline Unknown & $19(0.5 \%)$ & $11(0.6 \%)$ & + & + & + & + & \\
\hline \multicolumn{8}{|c|}{ Geographic (Census) region } \\
\hline Northeast & $530(13.1 \%)$ & ++ & ++ & ++ & ++ & ++ & $<.0001$ \\
\hline Midwest & 1068 (26.3\%) & $542(28.6 \%)$ & $128(24.9 \%)$ & $110(23.6 \%)$ & $130(24.0 \%)$ & $158(24.7 \%)$ & \\
\hline South & 1806 (44.5\%) & $829(43.8 \%)$ & $245(47.7 \%)$ & $225(48.3 \%)$ & $221(40.9 \%)$ & $286(44.7 \%)$ & \\
\hline West & $622(15.3 \%)$ & $261(13.8 \%)$ & 69 (13.4\%) & 70 (15.0\%) & $110(20.3 \%)$ & $112(17.5 \%)$ & \\
\hline Puerto Rico & $28(0.7 \%)$ & + & + & + & + & + & \\
\hline \multicolumn{8}{|c|}{ Total number of unique patients seen at oncology clinics at medical center } \\
\hline Mean (SD) & $217.7(157.2)$ & $226.0(159.5)$ & $218.2(157.5)$ & $204.1(149.9)$ & $196.5(150.4)$ & $220.3(158.9)$ & \\
\hline Median (Q1-Q3) & $\begin{array}{l}196.0(95.0- \\
302.0)\end{array}$ & $\begin{array}{l}208.0(99.0- \\
328.0)\end{array}$ & $\begin{array}{l}191.0(93.0- \\
301.0)\end{array}$ & $\begin{array}{l}185.5(90.0- \\
290.0)\end{array}$ & $\begin{array}{l}170.0(85.0- \\
275.0)\end{array}$ & $\begin{array}{l}204.0(93.5- \\
318.5)\end{array}$ & 0.0009 \\
\hline \multicolumn{8}{|c|}{ Total number of oncologists at medical center } \\
\hline Mean (SD) & $12.8(7.7)$ & $10.6(5.9)$ & $10.8(5.2)$ & $10.9(5.5)$ & $9.6(5.8)$ & $9.7(5.7)$ & \\
\hline Median (Q1-Q3) & $12.0(7.0-17.0)$ & $11.0(6.0-15.0)$ & $11.0(6.0-14.0)$ & $11.0(7.0-15.0)$ & $9.0(5.0-14.0)$ & $9.0(5.0-14.0)$ & $<.0001$ \\
\hline
\end{tabular}

+ Cell size $<11$ suppressed per VA policy

++ Suppressed cell size so one cannot calculate sample size for cells with $n<11$

${ }^{a} P$-value based on Chi-square tests for categorical variables and Kruskal-Wallis tests for continuous variables

${ }^{\mathrm{b}}$ Single or unknown marriage status not shown due to small cell sizes

\section{Discussion}

This study documents the most recent CCRT treatment patterns in a national cohort of VHA patients with unresectable stage III NSCLC in the United States. Our study found that among this cohort, the proportion of patients receiving CCRT increased slightly from 44\% in 2013 to 50\% in 2017. A previous study found that from 2001 to 2010, $59 \%$ of VHA patients who received RT and CT within 4 months of being diagnosed with unresectable, stage III NSCLC received CCRT as opposed to SCRT [13]. Our analysis of the 2013-2017 data showed that this proportion increased to $79 \%$, demonstrating that prescribers are following the increasing evidence that CCRT is more efficacious than SCRT [5-10].

However, among the whole cohort of VHA patients with unresectable stage III NSCLC, less than half (47\%) are receiving CCRT. After excluding those who received no treatment, approximately $55 \%$ received CCRT, which is similar to the $52 \%$ that was recently reported to have received CCRT in a US Medicare population (generally 
Table 2 Adjusted odds ratios for receipt of concurrent CRT versus any other treatment ${ }^{a}$

\begin{tabular}{|c|c|c|c|}
\hline \multirow{2}{*}{$\begin{array}{l}\text { Characteristic } \\
\text { Patient-related }\end{array}$} & \multirow[t]{2}{*}{ Estimate } & \multicolumn{2}{|c|}{$\begin{array}{l}95 \% \\
\text { Confidence } \\
\text { Limits }\end{array}$} \\
\hline & & Lower & Upper \\
\hline Female & 0.64 & 0.40 & 1.03 \\
\hline White & 1.24 & 1.004 & 1.53 \\
\hline Hispanic & 0.71 & 0.39 & 1.31 \\
\hline Married & 1.13 & 0.96 & 1.33 \\
\hline $\begin{array}{l}\text { Medicare enrollment any time between } \\
2013 \text { and } 2017\end{array}$ & 1.13 & 0.89 & 1.43 \\
\hline Medicaid-eligible & 0.63 & 0.34 & 1.14 \\
\hline \multicolumn{4}{|l|}{ Priority status (reference: non-service connected) } \\
\hline Service connected $\geq 50 \%$ & 1.12 & 0.94 & 1.33 \\
\hline Service connected $<50 \%$ & 1.01 & 0.81 & 1.27 \\
\hline Urban residence & 1.09 & 0.91 & 1.33 \\
\hline \multicolumn{4}{|l|}{ Histology (reference: squamous) } \\
\hline Adenocarcinoma & 0.91 & 0.77 & 1.07 \\
\hline Other & 0.80 & 0.62 & 1.02 \\
\hline \multicolumn{4}{|l|}{ Smoking status (reference: never) } \\
\hline Current smoker & 1.41 & 0.90 & 2.20 \\
\hline Former smoker & 1.34 & 0.85 & 2.10 \\
\hline Unknown & 1.13 & 0.65 & 1.95 \\
\hline \multicolumn{4}{|l|}{ Diagnosis year (reference: 2013) } \\
\hline 2017 & 1.65 & 1.27 & 2.16 \\
\hline 2016 & 1.36 & 1.06 & 1.76 \\
\hline 2015 & 1.11 & 0.88 & 1.39 \\
\hline 2014 & 1.22 & 0.98 & 1.53 \\
\hline Age (increment $=10$ ) & 0.67 & 0.60 & 0.76 \\
\hline $\begin{array}{l}\text { Charlson-Deyo comorbidity score } \\
\text { (increment }=1 \text { ) }\end{array}$ & 0.94 & 0.91 & 0.97 \\
\hline $\begin{array}{l}\text { Distance between patient residence and } \\
\text { medical center based on zip code } \\
\text { (increment }=100 \text { miles) }\end{array}$ & 0.95 & 0.84 & 1.08 \\
\hline \multicolumn{4}{|l|}{ Medical center-related } \\
\hline \multicolumn{4}{|l|}{ Geographic (Census) region (reference: South) } \\
\hline Northeast & 1.01 & 0.66 & 1.55 \\
\hline Midwest & 1.19 & 0.82 & 1.73 \\
\hline West & 0.89 & 0.60 & 1.33 \\
\hline $\begin{array}{l}\text { Medical Center Certified by Commission on } \\
\text { Cancer }\end{array}$ & 0.93 & 0.68 & 1.27 \\
\hline $\begin{array}{l}\text { Total number of unique patients seen at } \\
\text { oncology clinics at medical center of } \\
\text { diagnosis during diagnosis year } \\
\text { (increment }=100 \text { ) }\end{array}$ & 1.03 & 0.92 & 1.15 \\
\hline $\begin{array}{l}\text { Total number of oncologists at medical } \\
\text { center of diagnosis (increment }=10 \text { ) }\end{array}$ & 0.93 & 0.70 & 1.22 \\
\hline
\end{tabular}

at least 65 years of age and above) based on 2009 to 2014 data [18]. These rates are higher than reported rates outside the US. A study in China using 2013 to 2017 data reported CCRT use in $45 \%$ of 749 patients with unresectable stage III NSCLC treated at a single institution [25]. In the Netherlands, a multicenter retrospective study found that among 216 patients at least 70 years of age diagnosed with unresectable stage III NSCL C between 2009 and 2013, 33\% received CCRT [26]. In Turkey, a single-hospital study examining 130 patients at least 70 years of age with unresectable stage III NSCL C over 2005 to 2017 found that CCRT was used in $23 \%$ of patients [27].

Our results should be interpreted with an understanding of the VHA patient population, which is more likely to smoke currently or formerly compared to the general US population [28]. In our study cohort, 91\% were current (56\%) or former smokers (35\%), which was most similar to the Netherlands cohort (40\% current and 54\% former smokers) [26]. The high rate of smoking may be concerning since studies in stage III NSCLC patients indicate that current smoking is associated with poorer prognosis [29-31]. A recent study found that high-risk smokers (i.e., current or former smokers with a 30 or more pack-year smoking history) with lung cancer had shorter survival and poorer pulmonary function, as well as were more likely to have squamous histology [32]. In our study, $52 \%$ of patients had squamous histology which is higher than the $42 \%$ reported in the US Medicare population cohort. It is also higher than the 32 and $46 \%$ reported for the study cohorts in the Netherlands and Turkey, but lower than the 55\% reported for the study cohort in China [25-27]. In our study, neither smoking status nor squamous histology was associated with receipt of CCRT after adjusting for other patientand facility-level factors.

Patient factors that were associated with not receiving CCRT included increasing age and comorbidity burden, consistent with studies in US Medicare populations [18]. Chart review revealed that the majority of documented reasons for patients not receiving CCRT were related to not being a candidate due to frailty. This is aligned with a recent survey of US oncologists in which the most reported reason (64\%) for not recommending CCRT was that the patient would be unlikely to tolerate due to comorbidities, poor performance status, and/or advanced age [33]. Other reported reasons included patient preference (47\%), targetable mutation identified in the patient $(40 \%)$, ability of the patient to travel consistently to receive treatment (40\%), and cost (34\%). Similar to the number one reported reason, the most common motive in the Netherlands for not receiving CCRT was comorbidity and/or performance status (58\%) [26]. 
Guidelines agree that a patient's anticipated tolerance to therapy is an important factor when selecting treatment; however, studies have suggested that age alone is an insufficient reason to forego CCRT [10, 34, 35]. For example, a phase 3 randomized trial and its longtermfollow-up study assessed CCRT using low-dose carboplatin versus RT only in elderly patients ( $>70$ years) with unresectable NSCLC and found that in both the short-term and long-term, CCRT increased overall survival [35]. Real-world studies from the Netherlands and Turkey also suggest that CCRT use in older patients (70 years of age and above) is associated with better survival $[26,27]$. However, a study of VHA patients with NSCLC found that advancing age was a much stronger negative predictor of treatment receipt than comorbidity, contrary to evidence and guidelines stressing the importance of assessing comorbidity.

In addition to age and comorbidity burden, there were also differences in CCRT rates by race. In adjusted analyses, white patients were modestly more likely to receive CCRT compared to non-white patients. This is consistent with studies in US non-veteran populations that found that blacks, Asian-Pacific Islanders, and Hispanics were less likely receive CCRT compared to whites [18, 36]. Future studies should be performed to better understand these potential racial disparities and reasons for nonreceipt of CCRT so that effective interventions can be developed to address barriers to $\mathrm{NCCN}$ recommended care.

\section{Limitations}

Findings from this study should be interpreted in light of the limitations. We assessed treatment patterns in patients with unresectable, stage III NSCLC who were receiving their cancer care within VHA. Our results are not generalizable beyond this sample. Our treatment definitions were based on chemotherapy and radiation therapy start dates because the VA CRS did not systematically collect more detailed information regarding chemotherapy and radiation therapy administration for all patients. For example, if patients received their chemotherapy in VHA, but their radiation therapy in a non-VHA setting, only documentation of chemotherapy and radiation therapy start dates was required. However, we used previously published treatment definitions and performed multiple sensitivity analyses (e.g., varying time windows, examining rates among VHA facilities equipped to provide on-site radiation therapy services) to ensure that our estimates were robust. Additionally, our study period did not extend beyond 2017 because this was the most recent data available at the start of the study, given the time required for cancer registrars to carefully review and abstract data for the VA CRS. Our data also did not provide Eastern Cooperative Oncology Group performance status for all patients. Therefore, we calculated and adjusted for Charlson-Deyo comorbidity score, which could be determined for all patients.

\section{Conclusion}

CCRT rates among VHA patients with unresectable, stage III NSCLC slightly increased from 2013 to 2017; however in 2017, only half were receiving CCRT. Negative predictors included increasing age and comorbidity burden, while positive predictors included white race. Providers should consider comorbidity burden in addition to age, and be aware of potential racial disparities, when selecting among various therapeutic options. In chart review, reasons for patients not receiving CCRT were documented in less than half of patients. More research is needed to understand why eligible patients do not receive CCRT.

\section{Abbreviations}

aOR: Adjusted odds ratio; CDW: Corporate data warehouse; CRS: Cancer registry system; CCRT: Concurrent chemoradiation therapy; $\mathrm{Cl}$ : Confidence interval; CT: Chemotherapy; NSCLC: Non-small cell lung cancer; RT: Radiation therapy; SCRT: Sequential chemoradiation therapy; US: United States; VA: Department of Veterans Affairs; VHA: Veterans Health Administration

\section{Supplementary Information}

The online version contains supplementary material available at https://doi. org/10.1186/s12885-021-08577-y.

\section{Additional file 1: Appendix. International Classification of Diseases and Related Health Problems Codes for Lung Cancer. Appendix Table 2 Histology Codes for Non-small Cell Lung Cancer. Appendix Table 3a. CPT Codes for Lung Resection. Appendix Table 3b. ICD-9 Procedure Codes for Lung Resection. Appendix Table 3c. ICD-10 Procedure Codes for Lung Resection. Appendix Table 4. Stop Codes for VA Oncology Clinics. Appendix Table 5. Chemotherapy Drugs. Appendix Table 6. Radiation Therapy Codes. Appendix Table 7. Variation in Treatment Rates Across 108 Medical Centers. Appendix Table 8. Treatment Rates Based on Expanding Initial Treatment Time Window from 120 days to 180 days. Ap- pendix Table 9. Treatment Rates Based on Varying Time Window Be- tween CT and RT to Define CCRT. Appendix Table 10. Reasons for Not Receiving CCRT per Chart Review. Appendix Fig. 1. Total and Annual Treatment Rates in the Subgroup Seen at Medical Centers with Radiation Therapy $(n=2067)$. Appendix Fig. 2. Treatment Rates by Census Region in the Subgroup Seen at Medical Centers with Radiation Therapy $(n=2067)$. Appendix Fig. 3. Boxplot of Variation in Treatment Rates Across $38^{*}$ Medical Centers that Provide Radiation Therapy.}

\section{Acknowledgements}

Support for VA/CMS data provided by the Department of Veterans Affairs, VA Health Services Research and Development Service, VA Information Resource Center (Project Numbers SDR 02-237 and 98-004).

\section{Authors' contributions}

AH: Formal Analysis, Writing- Original Draft, Writing- Review \& Editing, Visualization, Project Administration; KML: Data Curation, Validation, WritingReview \& Editing; JAL: Conceptualization, Data Curation, Validation, WritingReview \& Editing; YL: Formal Analysis, Writing- Review \& Editing; PP: Validation, Writing- Review \& Editing; OVE: Data Curation, Validation, Project Administration; BJH: Data Curation, Validation; TB: Resources, Project Administration; CY: Resources, Project Administration; BS: Resources, Project Administration; MJK: Conceptualization, Writing- Review \& Editing; SDR: Conceptualization, Funding Acquisition, Supervision, Writing- Review \& Editing. All authors read and approved the final manuscript. 


\section{Funding}

This study was supported by a research agreement with AstraZeneca. The sponsor had no substantial role in study design; in the collection, analysis and interpretation of data; in the writing of the report; and in the decision to submit the article for publication.

\section{Availability of data and materials}

Data analyzed in the current study are not publicly available because they contain protected health information. These data can be made available from the corresponding author to credentialed VA clinicians and researchers on a written request and in accordance with VA regulations.

\section{Declarations}

\section{Ethics approval and consent to participate}

All methods were carried out in accordance with relevant guidelines and regulations. This study was approved by the ethics committees (institutional review boards) at Duke University, Durham VA, and VA Salt Lake City. The institutional review boards authorized a waiver of informed consent.

\section{Consent for publication}

Not applicable.

\section{Competing interests}

All authors report the research grant from AstraZeneca for this study. Dr. Kelley reports personal fees from Eisai, grants from Bavarian Nordic, grants from Novartis, grants from Bristol-Myers Squibb, grants from Regeneron, other from IBM, and personal fees from IBM Japan, outside the submitted work. Dr. Reed reports personal fees from AstraZeneca, outside the submitted work. Dr. Hung reports grants from PhRMA, and personal fees from Blue Cross Blue Shield Association and CVS Health outside the submitted work. Drs. Yong and Seal are employees of AstraZeneca. Dr. Buckingham was an employee of AstraZeneca during time of study and is currently employed by GSK

\section{Author details}

${ }^{1}$ Duke Clinical Research Institute, Duke University School of Medicine, Durham, NC, USA. ${ }^{2}$ Department of Population Health Sciences, Duke University School of Medicine, Durham, NC, USA. ${ }^{3}$ Department of Veteran Affairs Medical Center, Durham, NC, USA. ${ }^{4}$ Department of Veteran Affairs Salt Lake City Health Care System, Salt Lake City, UT, USA. ${ }^{5}$ Duke University Medical Center, Durham, NC, USA. ${ }^{6}$ AstraZeneca, Gaithersburg, MD, USA.

\section{Received: 10 March 2021 Accepted: 9 July 2021}

Published online: 16 July 2021

\section{References}

1. Dillman RO, Seagren SL, Propert KJ, Guerra J, Eaton WL, Perry MC, et al. A randomized trial of induction chemotherapy plus high-dose radiation versus radiation alone in stage III non-small-cell lung cancer. N Engl J Med. 1990; 323(14):940-5. https://doi.org/10.1056/NEJM199010043231403.

2. Le Chevalier T, Arriagada R, Quoix E, et al. Radiotherapy alone versus combined chemotherapy and radiotherapy in nonresectable non-small-cell lung cancer: first analysis of a randomized trial in 353 patients. J Natl Cancer Inst. 1991;83(6):417-23. https://doi.org/10.1093/jnci/83.6.417.

3. Dillman $\mathrm{RO}$, Herndon J, Seagren $\mathrm{SL}$, Eaton WL, Green MR. Improved survival in stage III non-small-cell lung cancer: seven-year follow-up of cancer and leukemia group B (CALGB) 8433 trial. J Natl Cancer Inst. 1996;88(17):1210-5. https://doi.org/10.1093/jnci/88.17.1210.

4. Sause W, Kolesar P, Taylor S IV, Johnson D, Livingston R, Komaki R, et al. Final results of phase III trial in regionally advanced unresectable non-small cell lung cancer: radiation therapy oncology group, eastern cooperative oncology group, and southwest oncology group. Chest. 2000;117(2):358-64. https://doi.org/10.1378/chest.117.2.358.

5. Curran WJ, Paulus R, Langer CJ, Komaki R, Lee JS, Hauser S, et al. Sequential vs. concurrent chemoradiation for stage III non-small cell lung cancer: randomized phase III trial RTOG 9410. J Natl Cancer Inst. 2011;103(19):145260. https://doi.org/10.1093/jnci/djr325.

6. Aupérin A, Le Péchoux C, Rolland E, et al. Meta-analysis of concomitant versus sequential radiochemotherapy in locally advanced non-small-cell lung cancer. J Clin Oncol. 2010;28(13):2181-90. https://doi.org/10.1200/JCO.2 009.26.2543.

7. Socinski MA, Rosenman JG, Halle J, Schell MJ, Lin Y, Russo S, et al. Doseescalating conformal thoracic radiation therapy with induction and concurrent carboplatin/paclitaxel in unresectable stage IIIA/B nonsmall cell lung carcinoma: a modified phase I/II trial. Cancer. 2001;92(5):1213-23. https://doi.org/10.1002/1097-0142(20010901)92:53.0.co;2-0.

8. Furuse K, Fukuoka M, Kawahara M, Nishikawa H, Takada Y, Kudoh S, et al. Phase III study of concurrent versus sequential thoracic radiotherapy in combination with mitomycin, vindesine, and cisplatin in unresectable stage III non-small-cell lung cancer. J Clin Oncol. 1999;17(9):2692-9. https://doi. org/10.1200/JCO.1999.17.9.2692.

9. O'Rourke N, Roqué I, Figuls M, Farré Bernadó N, Macbeth F. Concurrent chemoradiotherapy in non-small cell lung cancer. Cochrane Database Syst Rev. 2010;6. https://doi.org/10.1002/14651858.CD002140.pub3.

10. National comprehensive cancer network (NCCN). NCCN clinical practice guidelines in oncology. non-small cell lung cancer version 3.2020. 2020 feb 11;national comprehensive cancer network.

11. Ramnath N, Dilling TJ, Harris LJ, Kim AW, Michaud GC, Balekian AA, et al. Treatment of stage III non-small cell lung cancer: diagnosis and management of lung cancer, 3rd ed: American college of chest physicians evidence-based clinical practice guidelines. Chest. 2013;143(5 Suppl):e314S40S. https://www.nccn.org/professionals/physician_gls/pdf/nscl.pdf.

12. Handforth C, Clegg A, Young C, Simpkins S, Seymour MT, Selby PJ, et al. The prevalence and outcomes of frailty in older cancer patients: a systematic review. Ann Oncol. 2015;26(6):1091-101. https://doi.org/10.1 093/annonc/mdu540.

13. Veterans Health Administration. U.S. Department of Veterans Affairs website. Updated April 23, 2021. Accessed May 10, 2021. https://www.va.gov/health/.

14. Huang G, Muz B. U.S. Department of Veterans Affairs. 2017 Survey of veteran Enrollees' health and use of health care: data findings report. Published April 2018. Accessed May 1, 2021. https:/www.va.gov/HEALTHPOLICYPLANNING/ SOE2017NA_Enrollees_Report_Data_Findings_Report2.pdf.

15. Agha Z, Lofgren RP, VanRuiswyk JV, Layde PM. Are patients at veterans affairs medical centers sicker? A comparative analysis of health status and medical resource use. Arch Intern Med. 2000;160(21):3252-7. https://doi. org/10.1001/archinte.160.21.3252.

16. Zullig LL, Sims KJ, McNeil R, Williams CD, Jackson GL, Provenzale D, et al. Cancer incidence among patients of the U.S. veterans affairs health care system: 2010 update. Mil Med. 2017;182(7):e1883-91. https://doi.org/10.72 05/MILMED-D-16-00371.

17. Santana-Davila R, Devisetty K, Szabo A, Sparapani R, Arce-Lara C, Gore EM, et al. Cisplatin and etoposide versus carboplatin and paclitaxel with concurrent radiotherapy for stage III non-small-cell lung cancer: an analysis of veterans health administration data. J Clin Oncol. 2015;33(6):567-74. https://doi.org/10.1200/JCO.2014.56.2587.

18. Bobbili P, Ryan K, DerSarkissian M, et al. Predictors of chemoradiotherapy versus single modality therapy and overall survival among patients with unresectable, stage III non-small cell lung cancer. PLoS One. 2020;15(3). https://doi.org/10.1371/journal.pone.0230444.

19. Who is eligible for medicare? U.S. Department of Health and Human Services website. Updated September 11, 2014. Accessed April 24, 2021. https://www.hhs.gov/answers/medicare-and-medicaid/who-is-elibible-formedicare/index.html.

20. Eligibility. Medicaid.gov website. Accessed April 24, 2021. https://www. medicaid.gov/medicaid/eligibility/index.html.

21. VA priority groups. U.S. Department of Veterans Affairs website. Updated July 13, 2020. Accessed April 24, 2021. https://www.va.gov/health-care/ eligibility/priority-groups/.

22. 2021 VA health care copay rates. U.S. Department of Veterans Affairs website. Updated March 1, 2021. Accessed April 24, 2021. https://www.va. gov/health-care/copay-rates/.

23. Commission on Cancer. American College of Surgeons website. Accessed April 24, 2021. https://www.facs.org/quality-programs/cancer/coc.

24. Statistical Groupings of States and Counties. Accessed April 24, 2021. https://www2.census.gov/geo/pdfs/reference/GARM/Ch6GARM.pdf.

25. Zhang T, Bi N, Zhou Z, Chen D, Feng Q, Liang J, et al. The impact of age on the survival outcomes and risk of radiation pneumonitis in patients with unresectable locally advanced non-small cell lung cancer receiving chemoradiotherapy. J Thorac Dis. 2020;12(8):4347-56. https://doi.org/10.21 037/jtd-20-2137. 
26. Driessen EJ, Bootsma GP, Hendriks LE, van den Berkmortel FW, Bogaarts BA, van Loon JG, et al. Stage III non-small cell lung Cancer in the elderly: patient characteristics predictive for tolerance and survival of chemoradiation in daily clinical practice. Radiother Oncol. 2016;121(1):26-31. https://doi.org/10.1016/j.radonc.2016.07.025.

27. Sakin A, Sahin S, Atci MM, Sakin A, Yasar N, Geredeli C, et al. The effect of different treatment modalities on survival in elderly patients with locally advanced non-small cell lung cancer. Pulmonology. 2021;27(1):26-34. https://doi.org/10.1016/j.pulmoe.2019.11.007.

28. Odani S, Agaku IT, Graffunder CM, Tynan MA, Armour BS. Tobacco product use among military veterans - United States, 2010-2015. MMWR Morb Mortal Wkly Rep. 2018;67(1):7-12. https://doi.org/10.15585/mmwr. mm6701a2.

29. Bhatt VR, Batra R, Silberstein PT, Loberiza FR Jr, Ganti AK. Effect of smoking on survival from non-small cell lung cancer: a retrospective Veterans' Affairs central Cancer registry (VACCR) cohort analysis. Med Oncol. 2015;32(1):339. https://doi.org/10.1007/s12032-014-0339-3.

30. Ferketich AK, Niland JC, Mamet R, Zornosa C, D'Amico TA, Ettinger DS, et al. Smoking status and survival in the national comprehensive cancer network non-small cell lung cancer cohort. Cancer. 2013;119(4):847-53. https://doi. org/10.1002/cncr.27824.

31. Yano T, Miura N, Takenaka T, Haro A, Okazaki H, Ohba T, et al. Neversmoking nonsmall cell lung cancer as a separate entity: clinicopathologic features and survival. Cancer. 2008;113(5):1012-8. https://doi.org/10.1002/ cncr.23679.

32. Ban WH, Yeo CD, Han S, Kang HS, Park CK, Kim JS, et al. Impact of smoking amount on clinicopathological features and survival in non-small cell lung cancer. BMC Cancer. 2020;20(1):848. https://doi.org/10.1186/s12885-020-073 58-3.

33. Cotarla I, Boron ML, Cullen SL, et al. Treatment decision drivers in stage III non-small-cell lung cancer: Outcomes of a web-based survey of oncologists in the united states. JCO Oncol Pract. 2020:JOP1900781. https://doi.org/1 0.1200/JOP.19.00781.

34. Wang S, Wong ML, Hamilton N, Davoren JB, Jahan TM, Walter LC. Impact of age and comorbidity on non-small-cell lung cancer treatment in older veterans. JCO. 2012;30(13):1447-55. https://doi.org/10.1200/JCO.2011.39.52 69.

35. Atagi S, Kawahara M, Yokoyama A, Okamoto H, Yamamoto N, Ohe Y, et al. Japan clinical oncology group lung Cancer study group. Thoracic radiotherapy with or without daily low-dose carboplatin in elderly patients with non-small-cell lung cancer: a randomised, controlled, phase 3 trial by the Japan clinical oncology group (JCOG0301). Lancet Oncol. 2012;13(7): 671-8. https://doi.org/10.1016/S1470-2045(12)70139-0.

36. Miller ED, Fisher JL, Haglund KE, et al. Racial/ethnic disparities in the delivery of curative intent therapy in patients with stage III non-small cell lung cancer not treated surgically: An analysis of the national cancer database. Int J Radiat Oncol Biol Phys. 2018;102(3). https://doi.org/10.1016/j.jjrobp.201 8.07.1205.

\section{Publisher's Note}

Springer Nature remains neutral with regard to jurisdictional claims in published maps and institutional affiliations.

Ready to submit your research? Choose BMC and benefit from:
- fast, convenient online submission
- thorough peer review by experienced researchers in your field
- rapid publication on acceptance
- support for research data, including large and complex data types
- gold Open Access which fosters wider collaboration and increased citations
- maximum visibility for your research: over 100M website views per year
At BMC, research is always in progress.
Learn more biomedcentral.com/submissions

